\title{
PROGNOSTIC VALUE OF THE BONE TURNOVER MARKERS IN MULTIPLE MYELOMA
}

\author{
D. Auzina ${ }^{1,2, *}$, R. Erts ${ }^{3}$, S. Lejniece ${ }^{1,2}$ \\ ${ }^{1}$ Chemotherapy and Haematology Clinic, Riga East Clinical University Hospital, Riga LV 1079, Latvia \\ ${ }^{2}$ Department of Internal Diseases, Riga Stradins University, Riga LV 1007, Latvia \\ ${ }^{3}$ Department of Physics, Riga Stradins University, Riga LV 1079, Latvia
}

\begin{abstract}
Background: Multiple myeloma (MM) is characterized by osteolytic bone disease resulting from increased osteoclast activity and reduced osteoblast function. Aim: The aim of our research was to determine connection between bone turnover markers and presence of bone lesions, their degree of severity, to monitor MM bone disease and to assess effectiveness of anti-myeloma treatment. Materials and Methods: Serum samples and clinical data from 123 patients with newly diagnosed MM were collected at Riga East Clinical University Hospital (Riga, Latvia) from June 2014 to June 2016. Bone lesions detected by radiography, CT scans, MRI, and PET/CT were divided into degrees from 0 to $3(0-$ no bone involvement, $1-\leqslant 3$ bone lesions, $2-\geqslant 3$ bone lesions, $3-$ fracture). Staging was performed applying Durie/Salmon (DS) and International Staging System classifications. Progressive disease was defined as development of one or more new bone lesions. The levels of bone metabolic markers $\beta$-isomerized $\mathrm{C}$-terminal telopeptide of collagen type I ( $\beta$-CTX) and bone-specific alkaline phosphatase (bALP) were monitored regularly in the year. Results: Bone lesions were found in $86(69 \%)$ patients. From these $6(4 \%)$ patients had $1^{\text {st }}$ degree, $11(9 \%)$ had $2^{\text {nd }}$ degree and $69(56 \%)$ had $3^{\text {rd }}$ degree bone lesions. Level of the bone resorption marker $\beta$-CTX in the control group was $0.41 \mathrm{ng} / \mathrm{ml}$, which is lower than in MM patients $(\boldsymbol{p}<\mathbf{0 . 0 0 1})$. Spearman correlation coefficient analysis found a positive and statistically significant correlation $(\mathrm{rs}=\mathbf{0 . 5 1}, \boldsymbol{p}<\mathbf{0 . 0 0 1})$ between bone lesions degree and $\boldsymbol{\beta}$-CTX levels. Mean $\boldsymbol{\beta}$-CTX for patients without bone lesions was $0.72 \mathrm{ng} / \mathrm{ml}(\mathrm{SD}=\mathbf{0 . 6 4})$, but for patients with $3^{\text {rd }}$ degree bone lesions it was $1.34 \mathrm{ng} / \mathrm{ml}(\mathrm{SD}=0.65)$ difference being $38 \%$ $(p<0.001)$. In patients who responded to therapy after 6 months of treatment reduction of $\beta$-CTX was found compared to baseline values $(M=-0.65)$. In contrast, in patients who did not respond to therapy, there was a statistically significant $(p<0.001)$ increase in $\beta$-CTX values after six months of treatment compared to baseline values $(M=0.42)$. Exact cutoff value of $\beta$-CTX is 0.79 . When analyzing mean bALP, no significant difference between MM patients and control group was found. ANOVA statistical analysis showed no statistically significant differences in bALP levels at different degrees of bone lesions $(p=0.95)$ in MM patients. Analysis of bALP suitability as MM diagnostic marker using receiver operating characteristics curve showed that bALP is not applicable for clinical diagnosis of MM (AUC 0.5, $p>0.05$ ). However, $\beta$-CTX was found to be an excellent diagnostic marker for MM (AUC 0.91; 95\% confidence interval, 0.88-0.94; $p<0.001)$. Conclusions: Patients with MM and bone lesions have increased value of bone resorption marker $\beta$-CTX. There is a correlation between bone resorption marker and degree of bone lesions. Changes in $\beta$-CTX levels may be used to monitor the effectiveness of myeloma treatment.
\end{abstract}

Key Words: multiple myeloma, bone turnover markers, $\beta$-CTX, bALP.

Multiple myeloma (MM) is a B-cell malignancy characterized by proliferation of monoclonal plasma cells in the bone marrow. Bone lesions are a very common presenting feature in patients with $\mathrm{MM}$, with lytic bone destruction being a debilitating manifestation of the disease [1]. Bone disease is typical of MM and its occurrence increases with the progression of the disease. Bone disease can substantially affect patient morbidity and quality of life [1]. Most patients respond to initial treatment, but eventually almost all patients will have resistant relapse and die from the disease. Osteolytic lesions are seen in $70-80 \%$ of patients at diagnosis, while up to $90 \%$ develop lytic lesions during the course of their disease [2] and may cause skeletal-related event (SRE) with bone pain, pathological fractures, spinal cord compression, hypercalcemia and need to use radiation therapy or bone surgery [3],

Submitted: January 20, 2017.

*Correspondence: E-mail: dauzina@inbox.Iv

Abbreviations used: $\beta$-CTX $-\beta$-isomerized C-terminal telopeptide of collagen type I; bALP - bone-specific alkaline phosphatase; BTMs bone turnover markers; DS - Durie/Salmon; GFR - glomerular filtration rate; $\mathrm{MM}$ - multiple myeloma; SRE - skeletal-related event. but no tests have proven useful in identifying patients with increased risk for it.

$\mathrm{MM}$ is characterized by a tight relationship with the bone microenvironment. MM cells induce a significant alteration of the bone remodeling process due to the increase of osteoclast formation and activation and to the suppression of osteoblast differentiation leading to the development of osteolytic lesions [1, 4-7]. Biochemical markers of bone turnover may represent an alternative to evaluate the bone status of patients with myeloma. The activity of bone resorption and formation is reflected by bone turnover markers (BTMs) offering information about the ongoing activity in bone degradation and formation [8].

Bone markers are classified as resorption and formation markers. Bone resorption markers are the degradation products of osteoclasts or collagen degradation and bone formation markers are produced by osteoblastic cells or derived from procollagen metabolism. Bone resorption markers are degradation products of $\beta$-isomerized $C$-terminal telopeptide of collagen type I ( $\beta$-CTX), which constitutes $90 \%$ of the organic bone matrix and are highly specific for the degradation of type I collagen dominant in bone. 
Bone formation markers are products of osteoblast activity and include bone-specific alkaline phosphatase (bALP) representing membrane-bound osteoblast enzyme that is produced during bone formation. The aim of our research was to determine connection between BTMs and presence of bone lesions, their degree of severity, to monitor MM bone disease and to assess effectiveness of anti-myeloma treatment.

\section{MATERIALS AND METHODS}

Serum samples and clinical data from 123 patients with newly diagnosed MM were collected at Riga East Clinical University Hospital (Riga, Latvia) from June 2014 to June 2016. The patients enrolled in this study 70 (57\%) female and 53 (43\%) male had an age range of 33-86 years (median age was 67.65 years). Blood samples (and related data) were accessed after informed consent from all patients. Each patient's attending physician decided on therapy regardless of data being researched. Bone lesions at the time of diagnosis were detected using either conventional radiography, computed tomography (CT) scans, magnetic resonance imaging (MRI) or positron emission tomography (PET)/CT and were divided into degrees from 0 to $3(0-$ no bone involvement, $1-\leqslant 3$ bone lesions, $2-\geqslant 3$ bone lesions, 3 - fracture or vertebral collapse) according to [9]. Staging was performed applying Durie/Salmon (DS) and International Staging System classification system. Progressive disease was defined as development of one or more new bone lesions.

Treatment included cyclophosphamide, bortezomib, dexamethasone, erythropoietin, calcium, vitamin $\mathrm{D}$ and autologous stem cell transplantation. In addition, 4 patients were treated with low dose radiotherapy (up to $30 \mathrm{~Gy}$ ) in the course of treatment and 7 underwent vertebroplasty early in their treatment. All patients with bone disease (excluding patients with glomerular filtration rate (GFR) $<30 \mathrm{ml} / \mathrm{min}$ ) received zoledronate at a dose of $4 \mathrm{mg}$ every 4 weeks.

All analyzes were made within the same laboratory. Biochemical markers of bone remodeling, namely $\beta$-CTX and bALP were measured by electrochemiluminescence immunoassay ECLIA on Cobas analyzer (Roche Diagnostics, Germany) and chemiluminescence immunoassay on the LIAISON analyzer (DiaSorin, Inc., USA), respectively. Blood samples were collected at regular intervals (every 6 months over 1 year) at 7 o'clock in the morning from fasting patients. The samples were immediately centrifuged.

The control group comprised of 1128 age and sex matched patients. Excluded from the study were both MM patients and control group patients whose conditions creates primary or secondary osteoporosis or bone lesions, are receiving medications that could potentially lead to osteoporosis and patients with GFR $<30 \mathrm{ml} / \mathrm{min} / 1.73 \mathrm{~m}^{2}$. The study design, patients' information and consent forms were approved by the Ethic Committee of the Riga Stradins University and conducted according to the national ethical guidelines and the Helsinki Declaration.

Statistical analysis. IBM SPSS Statistics 23 software (IBM, USA) was used. Data were presented as mean $(M)$ and standard deviation $( \pm S D$ ) or median $(\mathrm{Me})$ and interquartile range (IQR) for continuous variables, and counts and percentages [\%] for categorical variables. Comparisons were made using $t$-test, Mann - Whitney test in case of non-normality and categorical variables were compared by Pearson's $\chi^{2}$ test. Changes in time were compared by using ANOVA for repeated measures. The relationships between variables were evaluated using Spearman Rank correlation coefficient (rs). Receiver operating characteristics (ROC) curve analysis was generated to test the predictive discrimination of MM patients with and without bone lesions. The area under each ROC curve (AUC) was calculated as a measure of overall diagnostic power. Sensitivity (Se), specificity (Sp), positive (PPV) and negative predictive values (NPV) were determined according to standard definitions. Exact cutoff values for bone markers were determined based on the best balance of Se and Sp. All tests were considered statistically significant at $p<0.05$.

\section{RESULTS AND DISCUSSION}

Bone lesions were found in $86(69.02 \%)$ patients. From these $6(4 \%)$ patients had $1^{\text {st }}$ degree, $11(9 \%)$ had $2^{\text {nd }}$ degree and 69 (56\%) had $3^{\text {rd }}$ degree bone lesions. Distribution between bone lesion localization was as follows: 100 patients had spinal lesions, 20 - costal, 16 - pelvic, 5-humeral, 4 - femoral and 3 - sternal. The most common combination of localizations were spinal with costal lesions together (84 patients). $82 \%$ of the vertebral fractures occurred in $T h_{V I I I}-L_{V}$ region of the spine and $50 \%$ of them found in the $\mathrm{Th}_{\mathrm{XI}}-\mathrm{L}_{1}$ region. In DS system stage 1, 2 and 3 were found in 24, 17 and 82 patients, respectively, but using International Staging System classification 57 patients had $1^{\text {st }}$ stage, $31-2^{\text {nd }}$ stage and $35-3^{\text {rd }}$ stage MM. Hypercalcemia was found in 16 (13\%) patients.

Statistical analysis showed that the average age of the control and study groups did not differ significantly ( $t$-test; $p>0.05$ ). Basic demographic indicators are shown in Table 1.

Table 1. Basic demographic indicators

\begin{tabular}{lccc}
\hline \multicolumn{1}{c}{ Indicators } & Control group & Research group & $p$ value \\
\hline Age (M \pm SD) & $65.01 \pm 9.14$ & $67.93 \pm 9.97$ & $>0.05$ \\
Female/male, $\mathrm{n} \mathrm{( \% )}$ & $846(75.0)$ & $70(56.9)$ & $>0.05$ \\
& $282(25.0)$ & $53(43.1)$ & \\
\hline
\end{tabular}

The $\beta$-CTX levels in the control group was $0.41 \mathrm{ng} / \mathrm{ml}$ $(S D=0.25)$, which is statistically significantly lower than in MM patients (Table 2). Spearman correlation coefficient analysis found that between bone lesions degree and $\beta$-CTX level there is a positive and statistically significant correlation ( $r s=0.51 ; p<0.001$ ). Mean $\beta$-CTX for patients without bone lesions was $0.72 \mathrm{ng} / \mathrm{ml}$ $(S D=0.64)$, but for patients with 3rd degree bone lesions it was $1.34 \mathrm{ng} / \mathrm{ml}(\mathrm{SD}=0.65)$ difference being $38 \%$. 
In patients who responded to therapy, reduction of $\beta$-CTX was found compared to baseline values $(\Delta M=$ $-0.65)$. In contrast, in patients who did not respond to therapy, there was an increase in $\beta$-CTX values after 6 months of treatment compared to baseline values $(\Delta M=0.42)$ ( $t$-test, $p<0.001)$. Value of $\beta$-CTX between $1^{\text {st }}$ and $2^{\text {nd }}$ collection of samples decreased in $62(71.26 \%)$ patients, but increased in 25 (28.74\%). ANOVA repeated measures shows statistically significant $\beta$-CTX decrease from the baseline value till $2^{\text {nd }}$ time of samples collection (after 6 months) $-58 \%$, but from $2^{\text {nd }}$ to $3^{\text {rd }}$ time (after a year of therapy) $-29.2 \%$ $(p<0.001)$.

Mean $\beta$-CTX value depending on the effect of therapy is shown in Table 3. $\beta$-CTX cutoff value for $\mathrm{MM}$ patients depending on gender and age is $0.79-0.87$ (Table 4). Exact cutoff value of $\beta$-CTX for all patients not adjusted for age and sex as diagnostic marker is $0.79 \mathrm{ng} / \mathrm{ml}$ ( $\mathrm{Sp}=91 \%$ (95\% Cl: 88-94); $\mathrm{Se}=$ $82 \%$ (95\% Cl: 69-85); PPV = 72\% (95\% Cl: 63-79); NPV $=93 \%(95 \% \mathrm{Cl}: 90-95))$. Only $42 \%$ of patients with no bone lesions have $\beta$-CTX value greater than 0.79 , while $94 \%$ of patients with $1^{\text {st }}$ to $3^{\text {rd }}$ degree bone lesions have $\beta$-CTX value greater than 0.79 .

Table 3. Changes of $\beta$-CTX value depending on the effect of therapy

\begin{tabular}{lcc}
\hline \multicolumn{1}{c}{ Patients } & $\begin{array}{c}\beta \text {-CTX M } \pm \text { SD } \\
(\min -\max )\end{array}$ & $\begin{array}{c}\text { Difference }(95 \% \\
\text { confidence inter- } \\
\text { val }-\mathrm{Cl}), p \text { value }\end{array}$ \\
\hline $\begin{array}{l}\text { Responding patients } \\
\text { Before treatment }(\mathrm{n}=114)\end{array}$ & $1.12 \pm 0.64(0.11-4.09)$ & $-0.65(0.41-0.66)$ \\
After 6 months therapy $(\mathrm{n}=84)$ & $0.47 \pm 0.36(0.03-1.77)$ & $p<0.001$ \\
Non-responding patients & & \\
Before treatment $(\mathrm{n}=9)$ & $1.38 \pm 0.74(0.86-3.22)$ & $0.42(0.21-0.62)$ \\
After 6 months therapy $(\mathrm{n}=9)$ & $1.80 \pm 0.92(1.10-3.98)$ & $p<0.001$ \\
\hline
\end{tabular}

When analyzing mean bALP, no significant difference between MM patients and control group was found. ANOVA statistical analysis showed no statistically significant differences in bALP levels at different degrees of bone lesions $(p=0.95)$ in MM patients. Value of bALP between $1^{\text {st }}$ and $2^{\text {nd }}$ collection of samples decreased in 60 (64.52\%) patients, but increased in 33 (35.48\%). Analysis of bALP suitability as MM diagnostic marker using ROC curve showed that bALP is not applicable for diagnosis (AUC $=0.5 ; p>0.05$ ). However, $\beta$-CTX was found to be an excellent diagnostic marker for MM (AUC $=0.91 ; p<0.001 ; 95 \% \mathrm{Cl}: 0.88-0.94$ ) (Figure).

Historically conventional radiography - wholebody X-rays - is the most common technique for the evaluation of bone disease in MM patients. However, whole-body X-rays has several limitations: it reveals Iytic disease when over $30 \%$ of the trabecular bone has been lost, while it cannot be used for the assessment of response to therapy and it has very low sensitivity for the pelvis and spine. Thus, whole-body low-dose CT can substitute conventional radiography as the standard technique for the evaluation of bone disease in MM. Furthermore, lytic bone lesions detected by CT and MRI have been included in the new criteria for the definition of symptomatic MM [10-13].
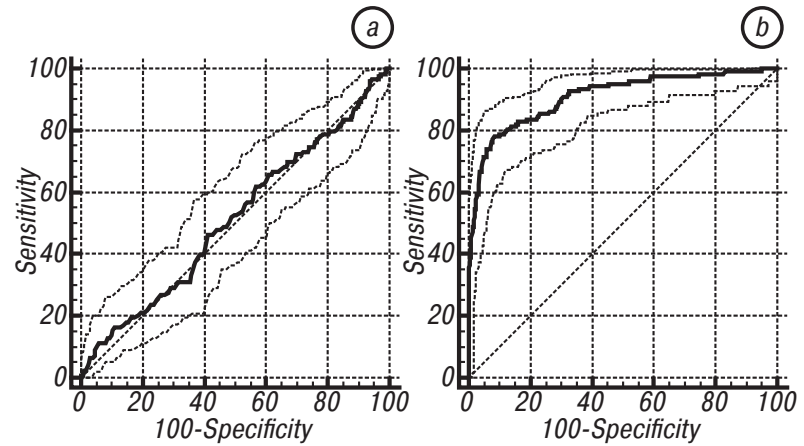

Figure. bALP (a) and $\beta-\mathrm{CTX}(b)$ ROC curves with $95 \% \mathrm{Cl}$ for all patients

The lesions rarely heal and bone scans are often negative in myeloma patients with extensive lytic lesions, offering very little in the follow-up of bone disease [14]. With this strategy, substantial damage may have occurred in bone before the patient becomes symptomatic and progressive bone disease is detected.

Biochemical markers are not harmful and are compatible with monthly monitoring. They have the potential to detect the destructive process as soon as it starts and before a lesion becomes detectable through conventional radiography [8, 15-18]. Data suggest that BTMs are useful prognostic factors that can predict patients' risk of SREs, bone lesion progression, and death. BTMs can also be used to measure to biologic effects of antiresorptive medications (bisphosphonates) and to identify subgroups of patients who are at high risk for disease progression and bone disease. BTMs could potentially be used as a tool for early diagnosis of bone lesions. Markers of bone metabolism should be incorporated into clinical practice as a tool to manage malignant bone disease of MM patients [3, 19-25].

\section{REFERENCES}

1. Sezer O. Myeloma bone disease: recent advances in biology, diagnosis, and treatment. Oncologist 2009; 14: 276-83.

Table 2. $\beta$-CTX and bALP levels in MM patients and control group with different degrees of bone lesions at the time of diagnosis

\begin{tabular}{|c|c|c|c|c|c|}
\hline Group & $\mathrm{n}$ & Median $\beta$-CTX (Q1-Q3) & $\beta-C T X M \pm S D(\min -\max )$ & Median bALP (Q1-Q3) & $\mathrm{bALP} M \pm \mathrm{SD}(\min -\max )$ \\
\hline Control group & 1128 & $0.37(0.23-0.56)$ & $0.41 \pm 0.25(0.03-2.50)$ & $11.40(8.80-15.80)$ & $13.80(3.90-217.00)$ \\
\hline MM patients & & & & & \\
\hline Bone lesion (degree - 0) & 39 & $0.61(0.48-0.96)$ & $0.72 \pm 0.64$ & $10.50(8.05-13.05)$ & $10.84 \pm 7.72$ \\
\hline Bone lesion (degree - 1) & 5 & $0.90(0.66-0.98)$ & $0.84 \pm 0.18$ & $11.80(11.10-12.65)$ & $11.86 \pm 0.95$ \\
\hline Bone lesion (degree - 2) & 11 & $0.89(0.86-1.03)$ & $0.99 \pm 0.28$ & $11.30(9.70-14.00)$ & $12.10 \pm 4.50$ \\
\hline Bone lesion (degree -3 ) & 68 & $1.11(0.96-1.50)$ & $1.34 \pm 0.65$ & $11.20(8.55-15.50)$ & $12.95 \pm 6.73$ \\
\hline
\end{tabular}

Table 4. $\beta$-CTX cutoff value for MM patients depending on gender and age at the time of diagnosis

\begin{tabular}{|c|c|c|c|c|c|c|c|}
\hline Gender of patients & Age of patients & AUC; $p$ value $(95 \% \mathrm{Cl})$ & Cutoff value & Se, $\%(95 \% \mathrm{Cl})$ & Sp, \% (95\% Cl) & PPV, \% (95\% Cl) & NPV, \% (95\% Cl) \\
\hline \multirow[t]{2}{*}{ Female } & $51-70$ & $0.91 ; p<0.001(0.85-0.97)$ & 0.79 & $80(63-91)$ & $92(79-88)$ & $58(43-71)$ & $97(94-99)$ \\
\hline & $>70$ & $0.87 ; p<0.001(0.79-0.96)$ & 0.82 & $68(56$ & $92(84-96)$ & $73(54-87)$ & $90(54-87)$ \\
\hline \multirow[t]{2}{*}{ Male } & $51-70$ & $0.92 ; p<0.001(0.84-0.99)$ & 0.79 & $75(53-90)$ & $93(82-98)$ & $85(63-96)$ & $88(76-96)$ \\
\hline & $>70$ & $0.80 ; p<0.001(0.67-0.94)$ & 0.87 & $62(40-81)$ & $85(87-98)$ & $88(63-98)$ & $57(34-78)$ \\
\hline
\end{tabular}


2. Terpos E, Morgan G, Dimopoulos MA, et al. International Myeloma Working Group recommendations for the treatment of multiple myeloma-related bone disease. J Clin Oncol 2013; 31: 2347-57.

3. Lipton A, Cook RJ, Coleman RE, et al. Clinical utility of biochemical markers of bone metabolism for improving the management of patients with advanced multiple myeloma. Clin Lymphoma Myeloma 2007; 7: 346-53.

4. Abe M. Targeting the interplay between myeloma cells and the bone marrow microenvironment in myeloma. Int J Hematol 2011; 94: 334-43.

5. Roodman GD. Osteoblast function in myeloma. Bone 2011; 48: 135-40.

6. Roodman GD. Pathogenesis of myeloma bone disease. Leukemia 2009; 23: 435-41.

7. Terpos E, Dimopoulos MA. Myeloma bone disease: pathophysiology and management. Ann Oncol 2005; 16: $1223-31$.

8. Lund T, Abildgaard N, Andersen TL, et al. Multiple myeloma: changes in serum C-terminal telopeptide of collagen type I and bone-specific alkaline phosphatase can be used in daily practice to detect imminent osteolysis. Eur J Haematol 2010; 84: 412-20.

9. Alexandrakis MG, Passam FH, Malliaraki N, et al. Evaluation of bone disease in multiple myeloma: a correlation between biochemical markers of bone metabolism and other clinical parameters in untreated multiple myeloma patients. Clin Chim Acta 2002; 325: 51-7.

10. D'Sa S, Abildgaard N, Tighe J, et al. Guidelines for the use of imaging in the management of myeloma. Br J Haematol 2007; 137: 49-63.

11. Waheed S, Mitchell A, Usmani S, et al. Standard and novel imaging methods for multiple myeloma: correlates with prognostic laboratory variables including gene expression profiling data. Haematologica 2013; 98: 71-8.

12. Coleman R, Body JJ, Aapro M, et al. Bone health in cancer patients: ESMO Clinical Practice Guidelines. Ann Oncol 2014; 25 (Suppl 3): 124-37.

13. Rajkumar SV. Updated diagnostic criteria and staging system for multiple myeloma. Am Soc Clin Oncol Educ Book 2016; 35: e418-23.
14. Terpos E. Biochemical markers of bone metabolism in multiple myeloma. Cancer Treat Rev 2006; 32 (Suppl 1): 15-9.

15. Clark RE, Flory AJ, Ion EM, et al. Biochemical markers of bone turnover following high-dose chemotherapy and autografting in multiple myeloma. Blood 2000; 96: 2697-702.

16. Hlaing TT, Compston JE. Biochemical markers of bone turnover - uses and limitations. Ann Clin Biochem 2014; 51 (Pt 2): 189-202.

17. Eom KS, Kim SJ, Lee JJ, et al. Changes in osteoblastic activity in patient who received bortezomib as second line treatment for plasma cell myeloma: a prospective multicenter study. Biomed Res Int 2014; 2014: 245-7.

18. Patel CG, Yee AJ, Scullen TA, et al. Biomarkers of bone remodeling multiple myeloma patients to tailor bisphosphonate therapy. Clin Cancer Res 2014; 20: 3955-61.

19. Peng $\mathrm{F}, \mathrm{Fu} \mathrm{R}$, Liu $\mathrm{H}$, et al. Clinical significance of serum bone metabolic markers in diagnosis and monitoring of myeloma bone disease. Zhonghua Yi Xue Za Zhi 2015; 95: 3436-9 (in Chinese).

20. Ting KR, Brady JJ, Hameed A, et al. Clinical utility of C-terminal telopeptide of type 1 collagen in multiple myeloma. Br J Haematol 2016; 173: 82-8.

21. Kyle RA, Yee GC, Somerfield MR, et al. American Society of Clinical Oncology 2007 clinical practice guideline update on the role bisphosphonates in multiple myeloma. J Clin Oncol 2007; 25: 2464-72.

22. Coleman R, Gnant M, Morgan G, Clezardin P. Effects of bone-targeted agents on cancer progression and mortality. J Natl Cancer Inst 2012; 104: 1059-67.

23. Pozzi S, Raje N. The role of bisphosphonates in multiple myeloma: mechanisms, side effects, and the future. Oncologist 2011; 16: 651-62.

24. Søe K, Delaissé JM, Jakobsen EH, et al. Dosing related effects of zoledronic acid on bone markers and creatinine clearance in patients with multiple myeloma and metastatic breast cancer. Acta Oncol 2014; 53: 547-56.

25. Lee J, Vasikaran S. Current recommendations for laboratory testing and use of bone turnover markers in management of osteoporosis. Ann Lab Med 2012; 32: 105-12. 